\title{
A variação das vogais médias pretônicas à luz das variáveis sociais em Porangatu e Jataí (GO)
}

\author{
The variation of the pretonic mid-vowels according to social \\ variables in Porangatu and Jatai (GO)
}

\author{
Daiane Silva Souza* \\ Universidade Federal da Babia \\ Salvador, Bahia, Brasil \\ Marcela Moura Torres Paim** \\ Universidade Federal da Babia \\ Salvador, Bahia, Brasil
}

\begin{abstract}
Resumo: Neste trabalho, apresenta-se uma análise do comportamento variável das vogais médias pretônicas em duas localidades do Estado de Goiás, observando como os fatores sociais atuam na seleção das variantes, condicionando a pronúncia das vogais como média-baixa (ex.: $\mathrm{t}[\mathcal{E}][[\mathcal{E}]$ visão e c[o]legas) ou como média-alta (ex.: b[o]rb[o]leta e t[e]rreno). Analisam-se amostras de duas localidades de Goiás — Porangatu e Jataí — coletadas a partir dos questionários fonético-fonológico e semântico-lexical (cf. Comitê Nacional, 2001). Os dados foram confrontados com os de Goiânia (GO). A análise tem como embasamento teórico os pressupostos da Sociolinguística Quantitativa. A partir do corpus observado, pode-se perceber que, nas localidades analisadas, há o predomínio das vogais-médias baixas. A variável sexo não se comportou como condicionante na escolha das variantes, mas expõe uma sutil tendência do sexo feminino no uso da vogal média-alta. A variável faixa etária se mostrou relevante na escolha das variantes. Os mais jovens expõem uma preferência pela média-alta, fato que levanta a discussão sobre a possível situação de mudança em curso em relação ao fenômeno analisado.
\end{abstract}

Palavras-chave: Vogais pretônicas. Variação. Sociolinguística. Projeto ALiB.

\begin{abstract}
In In this paper, we present an analysis of the variable behavior of pretonic mid-vowels in two localities of the state of Goiás, considering how social factors act in the selection of variants, conditioning the vowel pronunciation as open-medium vowels (for example: $\mathrm{t}[\mathcal{E}][[\mathcal{E}]$ visão and $\mathrm{c}[0]$ legas) or as mid-high vowel (for example: $\mathrm{b}[\mathrm{o}] \mathrm{rb}[\mathrm{o}]$ leta and $\mathrm{t}[\mathrm{e}] \mathrm{rreno}$ ). We analyze some samples from two localities of Goiás - Porangatu and Jataí - collected from the phonetic-phonological and semantic-lexical questionnaires (cf. Comitê Nacional, 2001). The data were confronted with data from Goiannia (GO). The analysis is based on a theoretical background from the Quantitative Sociolinguistics. From the corpus observed, we could realize the predominance of open-medium vowels in the localities analyzed. The sex variable did not behave as a conditioning factor in the choice of variants, but shows a slight tendency of female sex in the use of mid-high vowel. The age variable was relevant in the choice of variants. The younger ones exhibits medium-high preference, a fact that raises the discussion about the possible current situation of change in relation to the analyzed phenomenon.
\end{abstract}

Key-words: Pretonic vowels. Variation. Sociolinguistics. ALiB Project.

*Mestranda do Programa de Pós-Graduação em Língua e Cultura da Universidade Federal da Bahia. Salvador, Bahia, Brasil. E-mail: daianne.souza99@gmail.com.

** Doutora em Linguística pela Universidade Federal da Bahia, Professora Adjunto do Departamento de Letras Vernáculas do Instituto de Letras da Universidade Federal da Bahia. Salvador, Bahia, Brasil. E-mail: marcelamtpaim@yahoo.com.br. 


\section{INTRODUÇÃO}

É um consenso nas pesquisas existentes sobre as vogais pretônicas que "A língua portuguesa, na variedade brasileira, apresenta uma alternância fonética que caracteriza o falar de cada região." (SILVA, 1989, p.18). Os fenômenos fonéticos, no Português do Brasil, doravante $\mathrm{PB}$, são capazes de caracterizar as regiões dialetais no país. Neste trabalho, será analisada a realização variável das vogais médias em posição pretônica, sendo elas abertas, como em $t[\varepsilon][\varepsilon]$ fone e $\operatorname{tr}[0]$ vão ou fechadas, como t[e]l[e]fone e tr[o]vão, em Porangatu e Jataí, localidades de Goiás.

Desde a primeira proposta de divisão dialetal feita por Nascente em 1922, e reformulada em 1953, a pronúncia das vogais tem sido utilizada como principal parâmetro na distinção de áreas dialetais, Nascentes (1953, p. 19) afirma que "De um modo geral se pode reconhecer uma grande divisão: norte e sul [...]. No sul não há vogais protônicas abertas antes do acento (salvo determinados casos de derivação e a cadência é diferente da do norte."

As diferentes realizações das vogais médias já eram percebidas e distribuídas no meio geográfico de modo a caracterizar diferentes dialetos.

Na divisão de Nascentes, um dos critérios utilizado foi a existência das pretônicas abertas "em vocábulos que não sejam diminutivos nem advérbios em mente" (1953, p. 25). De acordo com essa divisão, Goiás apresenta áreas que fazem parte do falar do Norte, no subfalar nordestino (a parte de Goiás, que vai da serra do estrondo à nascente Parnaíba), e do falar do Sul, nos subfalares baiano (Goiás, parte que em da nascente do Paranaíba, seguindo pelas serras dos Javais, dos Xavantes, do Fanha e do Pilar, até a cidade de Pilas, rio das Almas, Pirenópolis, Santa Luzia e Arrependidos) e sulista (parte sul).

Considerando que em 1953, o Goiás ao qual Nascentes se referia, corresponde, atualmente, ao estado de Tocantins e ao atual Goiás. Com a organização atual, o estado ainda apresenta localidades que fazem parte do subfalar baiano, sulista e incaracterístico. No entanto, as localidades investigas pelo AliB que correspondem as estudadas por Nascentes estão distribuídas entre o falar baiano (Porangatu, São Domingos e Formosa) e o falar sulista (Goiás, Jataí, Quirinópolis, Catalão e Aruanã).

Vários estudos sobre as vogais já apontaram para o fato das sete vogais da língua portuguesa só se oporem em sílaba tônica ( $\mathrm{p}[\mathrm{o}] \mathrm{de}: \mathrm{p}[\mathrm{o}] \mathrm{de}$ ) e que, ao ocupar o lugar de pretônica, as vogais se reduzem a cinco, favorecendo a neutralização. Sendo assim, em posição pré-acentuada, as vogais médias abertas e fechadas não se opõem, se for dito $\mathrm{f}[\varepsilon]$ licidade ou f[e]licidade não haverá alteração no sentido.

Busca-se com o presente trabalho, analisar e descrever o fenômeno supracitado a partir dos pressupostos da Sociolinguística Quantitativa, dando ênfase aos fatores sociais que podem estar atuando na escolha das variantes. Ressaltando a importância de se considerar a língua como um uma estrutura multiforme e diversificada, Cardoso (1994, p.229) afirma que 
É preciso entender-se a natureza social da língua, a sua destinação histórica e conseqüentemente a sua estrutura imanente para chegar-se à compreensão dos fatos lingüísticos e a interpretação das mudanças que nela se operam.

Dessa forma, entende-se que a língua é antes de tudo um instrumento social, suas alterações ocorrem em um meio social, de interação e comunicação, e por isso, a organização da sociedade é importante para definir os processos de variação ou de mudança linguística. A fim de observar o quanto os fatores sociais condicionam a variação das pretônicas, o fenômeno será observado à luz do sexo e da faixa etária dos informantes. A amostra é constituída a partir do corpus do Projeto Atlas Linguístico do Brasil (Projeto ALiB).

\subsection{AS VARIÁVEIS SOCIAIS}

Desde o surgimento da Sociolinguística no século XX tem sido possível destacar as variáveis sociais que interferem na variação linguística e que em cada comunidade de fala esses fatores atuam de formas distintas. Para isso, Moreno Fernández (1998, p. 3334) aponta que existem dois motivos pelos quais as variáveis sociais devem ser estudadas, primeiro "porque los factores sociales actúan sobre la lengua de uma forma irregular", pois não há nada que condicione que a variação de um fenômeno deva ocorrer da mesma maneira em diferentes comunidades de fala, e segundo "porque los factores sociales no están configurados de forma idéntica em todas las comunidades".

As pesquisas sociolinguísticas buscam traçar o conjunto de regras variáveis que agem nos fenômenos em variação, para isso, analisam-se os fatores linguísticos combinados com as variáveis sexo, idade, escolaridade, entre outros, partindo da avaliação do falante sobre determinada variante, se é de prestígio ou não na comunidade observada. A avaliação do falante sobre o fenômeno linguístico constitui um dos cinco problemas da Sociolinguística.

O prestígio da variante pode determinar o grupo de falantes que a utiliza, por exemplo, os jovens que estão ingressando no mercado de trabalho e têm mais acesso à mídia, pode ser mais sensível ao uso da variante de prestígio.

Busca-se, neste trabalho, observar o quanto os fatores sociais influenciam no uso das vogais abertas e fechadas.

\section{METODOLOGIA}

A metodologia segue a do Projeto ALiB. Foram analisadas duas localidades interioranas do estado de Goiás, Porangatu (ponto 118) e Jataí (ponto 124).

Em cada localidade há quatro informantes, totalizando para o presente corpus oito informantes, quatro homens e quatro mulheres, distribuídos em duas faixas etárias, faixa I e faixa II. São organizados da seguinte maneira: 
Quadro 1: Faixa etária

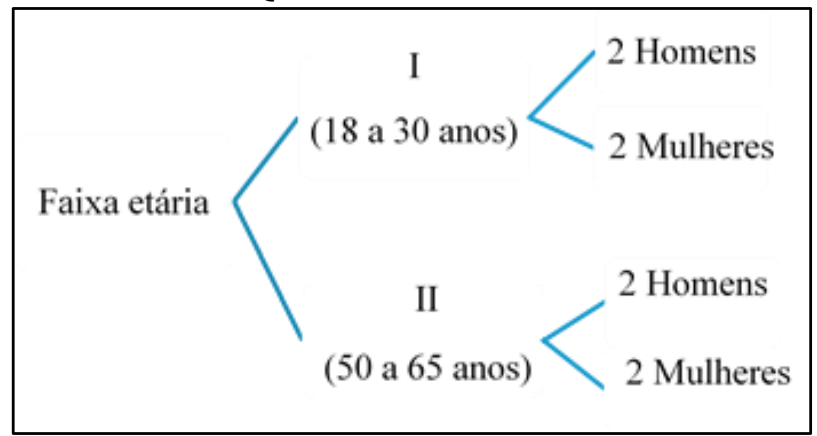

Fonte: Elaborado pelas pesquisadoras.

Foram utilizados os questionários Fonético-Fonológico e Semântico-Lexical. Validaram-se as respostas diretas e o discurso livre que ocorria entre elas, e foram descartados da análise os hiatos (ex.: moída), os ditongos (ex.: feitiço), as iniciais absolutas (ex.: elétrico) e os diminutivos (ex.: ovelhinha). Os dados foram obtidos a partir de questões como:

(QFF 152)

INQ.: Quando se quer saber uma coisa, se vai

INF.- Procurá [proku'ra].

INQ.- Quando se quer saber uma coisa.

INF.- Pergunta [peh'gũta].

INQ.- Então se vai...

INF.-Perguntar [pefigũ'ta].

Informante de Jataí, mulher, jovem (cf. Comitê... 2001, p. 18).

Após audição cuidadosa, os dados foram levantados, codificados e submetidos à análise estatística pelo programa ao GOLDVARB 2001. Foram consideradas as variáveis sexo e faixa etária. A escolaridade foi observada apenas na capital, devido a metodologia do ALiB, pois são analisados o nível universitário completo e fundamental incompleto nas capitais, e apenas fundamental incompleto nos interiores.

Os resultados referentes ao comportamento das vogais pretônicas na capital, Goiânia, já estão publicados no Atlas Linguístico do Brasil, e foram analisados por Mota e Lopes (2014), por isso, os resultados dos interiores (Porangatu e Jataî) serão comparados com os da capital, com o objetivo de analisar se os resultados das localidades interioranas vão refletir os aspectos encontrados na capital (Goiânia). Embora não seja possível comparar a escolaridade, essa variável será observada para se ter uma visão do comportamento desse fator. 


\subsection{AS LOCALIDADES}

Para este trabalho, foram selecionadas como amostra duas localidades de Goiás, Porangatu, que faz parte da mesorregião do norte do estado, e Jataí que faz parte da mesorregião do Sul.

Porangatu é considerado um dos mais influentes centros urbanos no Norte Goiano. O povoamento teve origem à época da descoberta do ouro, no século XVIII. Destaca-se como primeiros habitantes, a tribo dos "Canoeiros", e os ataques frequentes a essa tribo impediam o desenvolvimento naquela época.

Jataí tem sua história iniciada em 1836 quando uma família procedente de Minas Gerais entra nos sertões do sudoeste goiano pelo município de Rio Verde, formando uma fazenda de criação de gados às margens dos rios Claro e Ariranha. A base da economia atualmente é a agricultura e a pecuária. No ano de 1875, Jataí já possuía um respeitável comércio no setor da pecuária, juntamente com a atividade agrícola. Em 20 de fevereiro de 1890, a história de Jataí muda com a publicação do decreto n ${ }^{\circ} 22$ do governo da província, criando o município de Jataí e desligando-o de Rio Verde.

\section{DESCRIÇÃO E ANÁLISE}

Foram encontrados 738 (setecentos e trinta e oito) ocorrências, sendo 424 (quatrocentos e vinte e quatro) das vogais anteriores /E/ e 314 (trezentos e quatorze) das vogais posteriores $/ \mathrm{O} /$. Os resultados da distribuição total das vogais médias estão expostos na Tabela 1.

Tabela 1: Total de ocorrência vogais anteriores e posteriores em Porangatu e Jataí

\begin{tabular}{lll|lll}
\hline Variantes & Ocor. & $\%$ & Variantes & Ocor. & $\%$ \\
\hline [e] & $194 / 424$ & 46 & {$[0]$} & $141 / 314$ & 45 \\
{$[\varepsilon]$} & $230 / 424$ & 54 & {$[$ ग] } & $173 / 314$ & 55 \\
Total & 424 & 100 & Total & 314 & 100 \\
\hline
\end{tabular}

Fonte: Elaborada pelas pesquisadoras.

O que se pode observar é a maior frequência das vogais médias abertas. Para as anteriores $54 \%$ e para as posteriores $55 \%$. Esse resultado reflete o quadro encontrado na capital, Goiânia. Das capitais do Centro-Oeste, Goiânia foi a que apresentou menor índice de fechamento, como mostram Mota e Lopes (2014), das anteriores 67\% (0,29 P.R) e das posteriores, 61\% (0,24 P.R). 


\section{das Letras}

Tabela 2: Total de ocorrência vogais anteriores e posteriores em Goiânia

\begin{tabular}{lll|lll}
\hline Variantes & Ocor. & $\%$ & Variantes & Ocor. & $\%$ \\
\hline$[e]$ & $320 / 479$ & 67 & {$[0]$} & $237 / 388$ & 61 \\
\hline$\varepsilon]$ & $159 / 479$ & 33 & {$[$ ग] } & $151 / 388$ & 39 \\
\hline Total & 479 & 100 & Total & 388 & 100 \\
\hline
\end{tabular}

Fonte: Elaborada pelas pesquisadoras.

Observando as localidades separadamente, Porangatu apresenta 41\% e Jataí 50 $\%$ de frequência da média fechada anterior, e a frequência da média fechada posterior em Porangatu é de 43\% e em Jataí 47\%. Esses resultados podem apontar um indício de variação espacial, pois a localidade que faz parte da mesorregião norte apresenta menor frequência das variantes fechadas, enquanto a localidade situada ao sul, apresenta maior frequência.

\subsection{A VARIÁVEL SEXO}

A partir da Tabela 3 será observada a influência das variáveis sociais no uso das variantes médias fechadas.

Tabela 3: Vogais anteriores e posteriores segundo a variável sexo em Porangatu e Jataí.

\begin{tabular}{lll|ll}
\hline & & \multicolumn{2}{c}{ [o] } \\
\hline Sexo & Ocor. & $\%$ & Ocor. & $\%$ \\
\hline Homem & $94 / 227$ & 41 & $68 / 160$ & 42 \\
Mulher & $100 / 197$ & 50 & $73 / 154$ & 47 \\
\hline
\end{tabular}

Fonte: Elaborada pelas pesquisadoras.

A variável sexo não foi selecionada pelo Goldvarb. Essa variável não se comportou como condicionante na escolha das variantes nas localidades analisadas. Apesar disso, 
acredita-se ser importante expor esse resultado, pois os dados aparentemente sem significância também revelam alguma situação, segundo Guy e Zilles (2007, p. 215)

[...] há vários motivos que determinam um resultado sem significância. Um deles, obviamente, é que o possível efeito investigado realmente não exista no mundo. Mas também é possível que o efeito exista, mas que os dados coletados sejam insuficientes para revelá-lo.

Dessa forma, podemos refletir sobre a importância da variável sexo para a variação das vogais pretônicas, podem-se levantar duas hipóteses, os homens e as mulheres não apresentam diferenças quanto a escolhas das variáveis ou o corpus não foi suficiente para conseguir evidenciar essa diferença. Por isso, pretende-se ampliar o corpus futuramente, a fim de se ter uma visão mais abrangente desse panorama.

$\mathrm{Na}$ tabela 4 estão expostos os resultados das variantes fechadas na capital, Goiânia.

Tabela 4: Vogais anteriores e posteriores segundo a variável sexo em Goiânia

\begin{tabular}{llll|lll}
\hline & & & \multicolumn{3}{c}{ [o] } \\
\hline Sexo & Ocor. & $\%$ & P. R & Ocor. & $\%$ & P. R. \\
Homem & $143 / 231$ & 61 & 0,44 & $114 / 190$ & 60 & ----- \\
Mulher & $177 / 248$ & 71 & $\mathbf{0 , 5 5}$ & $123 / 198$ & 62 & ----- \\
\hline
\end{tabular}

Fonte: Elaborada pelas pesquisadoras.

O Goldvarb 2001 não selecionou a variável sexo nos interiores, no entanto pode-se notar uma pequena preferência no uso das variantes fechadas pelas mulheres. Quando verificado o contexto da capital, essa variável foi selecionada (para as vogais anteriores), as mulheres apresentam 0, 55 em P.R. O programa descartou essa variável em relação as posteriores, acredita-se que o questionário utilizado proporciona pouca ocorrência das posteriores, verifica-se um número menor de ocorrências em relação as anteriores.

Comparando os resultados dos interiores aos de Goiânia, é possível perceber que homens e mulheres se comportam de formas distintas em relação a esse fenômeno. Mesmo sendo uma diferença sutil, esses dados permitem que algumas reflexões sejam feitas, como a postura das mulheres em utilizar variantes que não sofrem estigma social.

Os trabalhos que consideram o sexo como variável social afirmam que quando a variante inovadora é estigmatizada há uma tendência das mulheres em ser conservadoras; em contrapartida, quando a variante inovadora não sofre estigma há uma tendência em implementar a mudança. 
Esse comportamento foi mostrado por Labov (1966), no estudo sobre o inglês de Nova York. A pronúncia retroflexa do -r pós-vocálico era a forma socialmente prestigiada, logo, se notou uma tendência das mulheres em liderar esse processo de mudança.

Antes de confirmar ou refutar essas afirmações, é preciso levar em consideração a estrutura social a qual pertencem os falantes, pois, se na comunidade, os papeis dos homens e das mulheres não apresentam diferenças significativas, se as mulheres estão imersas tanto quanto os homens no mercado de trabalho, é provável que o comportamento linguístico entre eles não apresente diferenças significativas; entretanto, se na comunidade homens e mulheres têm funções distintas, e há pouca possibilidade de mobilidade social para as mulheres, provavelmente essa distinção aparecerá no comportamento linguístico.

Moreno Fernández (1998, p. 39) afirma que “é evidente que não se pode oferecer uma explicação que seja igualmente válida para todas as comunidades”, confirmando a necessidade de relativização quando a variável sexo é analisada.

Outro aspecto que deve ser levado em consideração é a característica do fenômeno, pois se sabe que as diferenças entre homens e mulheres são maiores nos fatos linguísticos em que os falantes tem uma maior consciência, os quais as características podem "convertirse com más facilidad em marcas o símbolos sociales." (MORENO FERNÁNDEZ, 1998, p. 40).

Dessa maneira, é preciso avaliar que as localidades em análise fazem parte de uma cultura ocidental, cujo papeis sociais entre homens e mulheres são bem próximos, além do fenômeno não ser tão estigmatizado quanto os casos de concordância, por exemplo.

Ademais, transformações na organização social podem resultar na neutralização do efeito dessa variável. Com base nessas afirmações, é necessária a ampliação do corpus a fim de verificar se a atuação do sexo nas escolhas entre vogais abertas ou fechadas é neutralizada ou se o comportamento conservador das mulheres em relação a esse fenômeno irá se confirmar.

\section{2 A VARIÁVEL FAIXA ETÁRIA}

A idade dos falantes é constantemente observada nos estudos sociolinguísticos, pois "a idade condiciona a variação linguística com mais intensidade" e "sua realidade não é alterada por mudanças socioeconômicas, de atitudes ou de organização." (MORENO FERNÁNDEZ, 1998 p. 40).

Por meio da faixa etária é possível verificar se um fenômeno se encontra em variação estável ou se apresenta uma mudança em curso, o que permite essa observação é o método do tempo aparente.

Para Campoy e Almeida (2005, p. 42) uma das vantagens do estudo em tempo aparente é a "inmediatez de sus resultados y la mayor homogeneidad de la planificación y del desarrollo del estudio.”. Por meio desse tipo de método não há necessidade de esperar anos para verificar o que ocorrerá com determinado fenômeno. Sobre a observação de faixas etárias, Moreno Fernández (1998, p. 44) assinala que 


\section{das Letras}

El dessarrollo del cambio, observado em um momento determinado y em hablantes de generaciones distintas, oferece una imagen dinámica en $<<$ tiempo aparente $>>$ que permite proyectar cómo será esse cambio en el futuro, conforme vaya transcurriendo el $<<$ tiempo real $>>$.

Logo, a observação em tempo aparente permite que se faça uma projeção do que ocorrerá com o fenômeno linguístico no futuro.

Nas tabelas 5 e 6 são apresentados os resultados das variantes médias fechadas nos interiores e na capital, respectivamente, partir das faixas etárias. Lembrando que a faixa I é composta por falantes entre 18 e 30 anos e faixa II por falantes entre 50 e 65 anos.

Tabela 5: Vogais [e] e [o] segundo a variável faixa etária em Porangatu e Jataí.

\begin{tabular}{lccc|ccc}
\hline \multicolumn{3}{c|}{ [e] } & \multicolumn{3}{c}{ [o] } \\
\hline Faixa Etária & Ocor. & $\%$ & P. R. & Ocor. & $\%$ & P. R. \\
Faixa I & $102 / 197$ & 51 & $\mathbf{0 , 5 6}$ & $83 / 164$ & 50 & $\mathbf{0 , 5 5}$ \\
Faixa II & $97 / 227$ & 40 & 0,44 & $58 / 150$ & 38 & 0,43 \\
\hline
\end{tabular}

Fonte: Elaborada pelas pesquisadoras.

Tabela 6: Vogais [e] e [o] segundo a variável faixa etária em Goiânia

\begin{tabular}{lccc|ccc}
\hline \multicolumn{3}{c|}{ [e] } & \multicolumn{3}{c}{ [o] } \\
\hline Faixa Etária & Ocorrência & $\%$ & P. R. & Ocorrência & $\%$ & P. R. \\
Faixa I & $155 / 216$ & 71 & $\mathbf{0 , 5 5}$ & $123 / 178$ & 69 & $\mathbf{0 , 5 7}$ \\
Faixa II & $165 / 263$ & 62 & 0,45 & $114 / 210$ & 54 & 0,43 \\
& & & & & & \\
\hline
\end{tabular}

Fonte: Elaborada pelas pesquisadoras.

A faixa etária foi selecionada como condicionante no uso das variáveis, tanto nos interiores quanto na capital. A faixa I, mais jovem, utiliza com mais frequência as variantes de prestígio [e] e [o]. 
Esse resultado pode evidenciar que o uso das variantes fechadas está em situação de mudança em curso, pois os falantes mais jovens as utilizam com maior frequência, enquanto os mais velhos utilizam as variantes abertas. No entanto, essa afirmação deve ser feita com cautela, pois, para se obter uma projeção mais completa seria necessária uma faixa etária intermediária que se distinguisse das extremidades. O que esse resultando nos revela é que os mais jovens estão com uma maior tendência a utilizar as variantes fechadas, as quais são características dos grandes centros de difusão cultural e veiculadas nas mídias do Brasil.

\subsection{A VARIÁVEL ESCOLARIDADE}

A escolaridade foi considerada apenas na capital, e está presente neste estudo por mostrar um comportamento importante desse fator em relação as vogais médias.

Tabela 7: Vogais [e] e [o] segundo a variável escolaridade em Goiânia

[e] [o]

\begin{tabular}{lllll}
\hline Escolar. & Ocor. & $\%$ & Ocor. & $\%$ \\
\hline Fundamental & $138 / 214$ & 64 & $107 / 178$ & 60 \\
& & & & \\
Universitário & $182 / 265$ & $\mathbf{6 8}$ & $130 / 210$ & $\mathbf{6 1}$ \\
\hline
\end{tabular}

Fonte: Elaborada pelas pesquisadoras.

A escolaridade não foi selecionada como relevante pelo programa estatístico, e quase não há diferença percentual entre o nível fundamental e o universitário, como está exposto na Tabela 7.

Sendo assim, pode-se pensar na categorização feita por Votre sobre distinções presentes na dinâmica social e que interagem na escola (2015, p. 53), uma dessas categorias é composta pelos "fenômenos controlados pela escola, contra aqueles que não são objeto da atenção disciplinadora e gramaticizadora da mesma." Por exemplo, A escola pune formas como framengo e pobrema, mas é conivente com formas redundantes, como há anos atrás.

Dessa forma, é possível perceber que a escola regula com força alguns tipos de variação, como os exemplos citados de rotacismos, fazendo com que os falantes com mais tempo de escolaridade se monitore, e barre o uso das variantes estigmatizadas; em contrapartida, há casos de variação em que a escola não regula, pois não é tão evidente e não possui o mesmo grau de estigma, acredita-se que esse seja o caso das vogais médias pretônicas. Como não são tão perceptíveis como o rotacismo, por exemplo, a escola não pune o uso de uma variante ou outra, fazendo com que os falantes com o fundamental incompleto e o universitário completo não se distanciem no uso dessas variantes. 


\section{CONSIDERAÇÕES FINAIS}

Neste trabalho, pode-se refletir sobre o papel das variáveis sociais sobre o comportamento das vogais médias pretônicas em Goiás, utilizando como amostra, duas localidades interioranas, Porangatu e Jataí. Foi encontrado um total de 738 (setecentos e trinta e oito) ocorrências - 424 (quatrocentos e vinte e quatro) das vogais anteriores e 314 (trezentos e quatorze) das vogais posteriores. Essa amostra permitiu observar que houve maior ocorrência das vogais médias abertas nas localidades investigadas. Ademais, pode-se perceber que os fatores sociais são importantes para a compreensão do fenômeno.

Assim, o trabalho mostra, principalmente com a faixa etária, que o comportamento linguístico pode ser condicionado por situações extralinguísticas.

Pretende-se, futuramente, ampliar o corpus apresentado, a fim de complementar as informações aqui presentes, e corroborar os resultados encontrados. Além disso, outros estudos podem complementar estas informações, para que dessa forma seja possível descrever a realidade linguística do Brasil.

\section{REFERÊNCIAS}

CÂMARA JÚNIOR, Joaquim Mattoso. Estrutura da língua portuguesa. 42.ed. Petrópolis, RJ: Vozes, 2009 [1970].

CAMPOY, Juan Manoel; ALMEIDA, Manuel. Metodología de la investigación sociolinguística. Mágala: Editorial Comares, 2005.

CARDOSO, Suzana. Língua: meio de opressão ou de socialização. In: FERREIRA, Carlota et al. Diversidade do Português do Brasil: estudos de dialectologia rural e outros. 2. ed. Revista. Salvador: Centro Editorial e Didático da UFBA, 1994. p. 229-233.

COMITÊ NACIONAL DO PROJETO ALiB. Atlas lingüistico do Brasil: questionários 2001. Londrina: EDUEL, 2001.

GYE, Gregory Riordan; ZIILLES, Ana. Sociolinguística Quantitativa. São Paulo: Parábola Editorial, 2007. p. 214-217.

INFORMAÇÕES SOBRE JATAÍ. Disponível em: $<$ http:/ / cidades.ibge.gov.br/xtras/perfil.php?lang=\&codmun $=521190 \&$ search $=$ goias $\mid j$ atai. Acesso em 28 de maço de 2017.

INFORMAÇÕES SOBRE PORANGATU. Disponível em: $<$ http:/ / cidades.ibge.gov.br/painel $/$ historico.php?lang $=\&$ codmun $=521800 \&$ search $=$ go ias | porangatu | infograficos:-historico. Acesso em: 28 de março de 2017.

LABOV, William. The social stratification of English in New York. Washington, D.C.: Center for Applied Ligustics, 1996.

MORENO FERNÁNDEZ, Francisco. Princípios de sociolinguística y sociologia del linguaje. Barcelona: Editorial Ariel, 1998. p. 33-69. 
MOTA, Jacyra Andrade; LOPES, Paulo Henrique de Souza. Vogais médias pretônicas nas capitais brasileiras, com base nos dados do Atlas linguístico do Brasil (ALiB), 2014. Entregue para publicação.

NASCENTES, Antenor. O linguajar carioca. 2. ed. Rio de Janeiro: Organizações Simões, 1953. 217p.

ROBINSON, John; LAWRENCE, Helen; TAGLIAMONTE, Sali. Goldvarb 2001: a multivariate analysis application for Windows. Nova York: University of York, 2001.

SILVA, Myrian Barbosa da. As pretônicas no falar baiano. 377 f. (Tese de Doutorado em Língua Portuguesa) - Faculdade de Letras, Universidade Federal do Rio de Janeiro. Rio de Janeiro, semestre de 1989. Rio de Janeiro, 1989.

Recebido em: 05/06/2018

Aprovado em: 23/07/2018

Publicado em: 31/12/2018 